\title{
A Minimal Model for Gene Expression Dynamics of Bacterial Toxin-antitoxin Systems
}

Kosmas Kosmidis ( $\sim$ kosmask@auth.gr)

Aristotle University of Thessaloniki

Marc-Thorsten Hutt

Jacobs University Bremen

\section{Research Article}

Keywords: Toxin-antitoxin (TA) , physiology, Gene Expression, Bacterial Toxin-antitoxin Systems

Posted Date: June 25th, 2021

DOI: https://doi.org/10.21203/rs.3.rs-646336/v1

License: (a) This work is licensed under a Creative Commons Attribution 4.0 International License. Read Full License 


\title{
A minimal model for gene expression dynamics of bacterial toxin-antitoxin systems
}

\author{
Kosmas Kosmidis ${ }^{1, *}$ and Marc-Thorsten Hütt ${ }^{2}$ \\ ${ }^{1}$ Physics Department, Aristotle University of Thessaloniki, 54124 Thessaloniki, Greece \\ ${ }^{2}$ Department of Life Sciences and Chemistry, Jacobs University Bremen, 28759 Bremen, Germany \\ *kosmask@auth.gr
}

\begin{abstract}
Toxin-antitoxin (TA) modules are part of most bacteria's regulatory machinery for stress responses and general aspects of their physiology. Due to the interplay of a long-lived toxin with a short-lived antitoxin, TA modules have also become systems of interest for mathematical modelling. Here we resort to previous modelling efforts and extract from these a minimal model of TA dynamics on a timescale of hours, which can be used to describe time courses derived from gene expression data of TA pairs. We show that this model provides a good quantitative description of TA dynamics for the 11 TA pairs under investigation here, while simpler models do not.

Our study brings together aspects of Biophysics with its focus on mathematical modelling and Computational Systems Biology with its focus on the quantitative interpretation of 'omics' data.

This mechanistic model serves as a generic transformation of time course information into kinetic parameters. The resulting parameter vector can, in turn, be mechanistically interpreted. We expect that TA pairs with similar mechanisms are characterized by similar vectors of kinetic parameters, allowing us to hypothesize on the mode of action for TA pairs still under discussion.
\end{abstract}

\section{Introduction}

The vast majority of free-living bacteria contain a number of toxin-antitoxin (TA) gene pairs ${ }^{1-4}$. The toxin products target key cellular functions inhibiting cell growth and eventually leading to cell death, while the corresponding antitoxin neutralizes the toxin's effect, thus, forming a toxin-antitoxin system whose accurate expression regulation is vital to the survival of the cell ${ }^{5}$. These TA systems are currently classified in six groups (types I, II, III, IV, V, VI) ${ }^{2}$ according to the mechanism used by the antitoxin to neutralize the toxin. Types I-III are considered to be well-established TA systems ${ }^{3,6-9}$ while types IV-VI consist of newly discovered types ${ }^{10-14}$. Type II TA systems are the largest and best studied TA system class. Type II antitoxins are proteins. They typically have two domains, one that binds DNA and a second that binds and inhibits the activity of the cognate protein $\operatorname{toxin}^{2,3,9}$. The presence of toxin-antitoxin systems is considered to be associated to persistence, i.e. the multidrug tolerance of bacteria, which obviously compromises the effectiveness of antibiotics on many pathogenic bacteria ${ }^{15}$. It is believed ${ }^{4,15,16}$ that when antibiotics are applied, a small sub-population of bacteria, called persisters, enters a dormant, non-dividing state and thus are protected from being killed. Experiments have shown a connection between persister formation and the competition between a toxin and its antitoxin inside an E. coli cell. Toxins inhibit cell growth and most antibiotics target the cell during the growth phase. Cells entering this persistent state seem to be immune to antibiotics but this immunity is different from the one obtained through advantageous mutations that result in antibiotic resistance since it is not permanent or inherited ${ }^{17}$. Knowledge about toxin-antitoxin systems in bacteria is still accumulating ${ }^{18}$. This is true for the discovery of new toxin-antitoxin modules ${ }^{19}$, their classification ${ }^{5,20}$, their functional roles ${ }^{21,22,25,26}$ as well as their detailed molecular mechanisms ${ }^{27}$.

In E. coli, there are more than ten well-characterized type II TA systems ${ }^{1}$. These include relE-relB, yafQ-dinJ, yoeB-yefM, hipA-hipB, yafO-yafN, hicA-hicB, higB-higA, ypjF-yfjZ, mqsR-mqsA, ymcE-gnsA and ydaT-ydaS. ${ }^{10,28-37,57}$. The genomic location of each of these TA systems is indicated in Figure 1. It is of considerable practical importance to understand the dynamics of toxin-antitoxin systems and several plausible models for toxin-antitoxin dynamics and persister formation have been proposed (see, for example ${ }^{38-40}$ and references therein). It is also important that the proposed model predictions are compared to, nowadays available, high-throughput data. In this paper, we present a minimal model for the description of toxin-antitoxin type II dynamics in E. coli. The basic characteristics of the minimal model is that it assumes: (a) regulation of toxin and antitoxin production rate by means of a negative feedback through DNA binding of the toxin-antitoxin complex (b) toxin induced growth rate modulation. The model's predictions are compared to the RNA-Seq gene expression data published in $^{41}$ (see Results and Discussion).

Toxin-antitoxin dynamics have been of interest to mathematical modelling for a long time. So far, the focus of research has been on the basic dynamical properties of TA modules ${ }^{39,40,42,43}$ and the synchronization of multiple TA modules in response to 


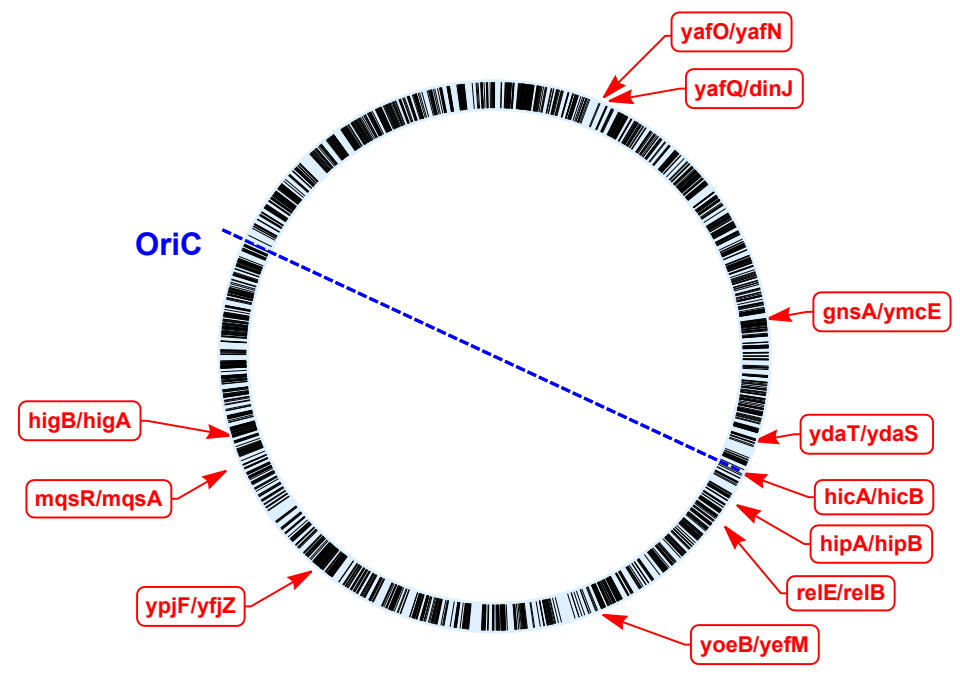

Figure 1. Genomic locations of the 11 TA modules studied in our investigation. Each gene is depicted as a radial dash on the circular chromosome. The location of each TA module is labelled with a red box. The OriC-Ter axis (from the origin of replication to the terminus of replication) is indicated for reference as a dashed blue line.

environmental stimuli (e.g., ${ }^{44}$ ), rather than the agreement with high-throughput data. For high-throughput data, in particular gene expression patterns, the dominant avenue of research has been to compare these patterns with large-scale regulatory networks or classes of regulatory mechanisms. In the case of bacterial gene regulation, successes have been understanding and experimentally confirming the role of small regulatory devices like feedforward loops ${ }^{45,46}$, the discovery of an interplay the regulatory network and chromosomal structure ${ }^{47-50}$ and the organization of gene expression along the axis from the origin (OriC) to the terminus (Ter) of replication ${ }^{50}$.

Toxin-antitoxin systems are often embedded in an intricate network of regulatory processes ${ }^{5}$ and part of functional regulatory modules ${ }^{51}$. There is evidence of collective behaviors arising from the interplay between toxin-antitoxin systems. Such a model of coupled toxin-antitoxin systems has for example been studied $i^{21}$ and $i^{44}$. Simple ODE models of (type-II) toxin-antitoxin systems have for example been formulated $\mathrm{in}^{21}$ with an emphasis on coupled systems and the spontaneous switching occurring in stochastic dynamics, $\mathrm{in}^{40}$, where conditional cooperativity of the RelBE system has been studied and its response to environmental stimuli (e.g., nutritional stress), in ${ }^{53}$, which contains a simplified system capable of excitable dynamics, as well as in ${ }^{39}$ and $^{44}$ with a focus on bistability. For type-I TA systems, a mathematical model has been developed in $^{54}$, offering insight in time scales involved.

Here we study the long-term dynamics of toxin-antitoxin pairs in time-resolved RNA-Seq data for E. coli. Our question is, whether the dynamics of all TA pairs in the data can be described by the same model, or whether qualitatively different models have to be assumed for the different TA modules.

\section{Methods}

Figure 2 shows a schematic of the basic characteristics of the minimal model of type II toxin-antitoxin gene expression. Toxin $T$ and antitoxin $A$ are expressed by neighbouring genes. It is known ${ }^{1,39}$ that toxins are more stable than the antitoxins, thus, the latter have to be constantly expressed in order to neutralize the toxin effects. The toxin and antitoxin form a complex $A T$ which inhibits toxin and antitoxin production. More complex toxin-antitoxin interaction (such as conditional cooperativity 39,40 or cooperation between multiple toxin-antitoxin systems ${ }^{17}$ ) are not included in the minimal model. Moreover, the presence of toxin has an inhibitory effect on the cell growth. This last fact is found to be an essential characteristic of an acceptable minimal model.

We denote the concentration of the antitoxin $A$ with the variable $y_{1}$, that of the toxin $T$ with $y_{2}$ and, finally, the concentration of the toxin-antitoxin complex $A T$ with $y_{3}$. The system of ordinary differential equations (ODEs) that describes the system is: 


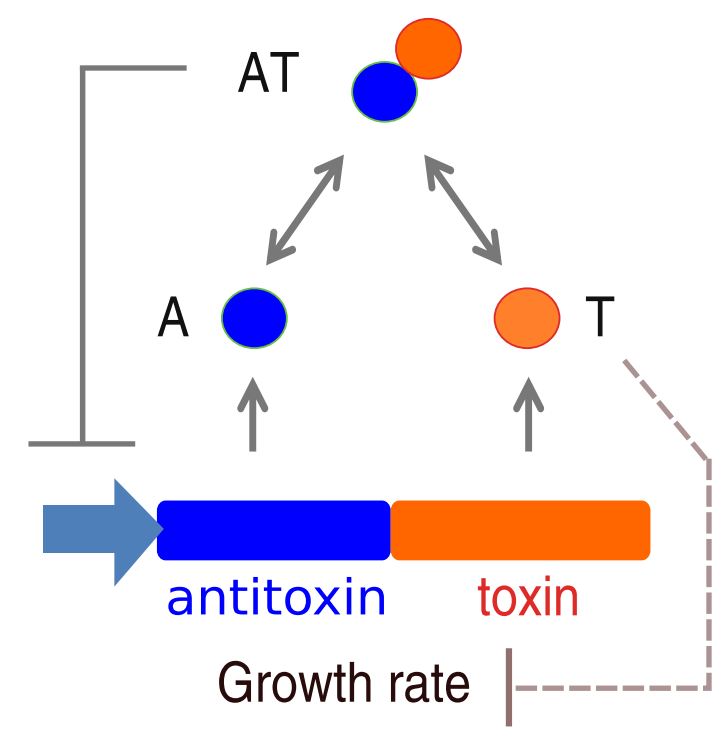

Figure 2. Schematic overview of the minimal model. Toxin $T$ and antitoxin $A$ are expressed by neighbouring genes. A toxin-antitoxin complex molecule $A T$ can be formed from one toxin and one antitoxin molecule. The $A T$ molecule down-regulates $A$ and $T$ production. At the same time toxin molecules inhibit cell growth.

$$
\begin{aligned}
\frac{d y_{1}}{d t} & =\frac{k_{1}^{\prime}}{\left(1+\frac{y_{3}}{s_{1}^{\prime}}\right)\left(b_{m}^{\prime} y_{2}+1\right)}-d_{1} y_{1}+d_{3} y_{3}-k_{3} y_{1} y_{2} \\
\frac{d y_{2}}{d t} & =\frac{k_{2}^{\prime}}{\left(1+\frac{y_{3}}{s_{2}^{\prime}}\right)\left(b_{m}^{\prime} y_{2}+1\right)}-\frac{d_{2} y_{2}}{b_{c}^{\prime} y_{2}+1}+d_{3} y_{3}-k_{3} y_{1} y_{2} \\
\frac{d y_{3}}{d t} & =-d_{3} y_{3}+k_{3} y_{1} y_{2} .
\end{aligned}
$$

Equation (3) is a standard chemical kinetics equation. We assume that the production rate of the complex $y_{3}$ is proportional to the product of the concentrations of $y_{1}$ and $y_{2}$, thus the term $k_{3} y_{1} y_{2}$ where $k_{3}$ is the respective rate constant. We also assume that the complex degrades to its constituents $A$ and $T$ with a rate constant $d_{3}$.

The inhibitory action of the $A T$ complex is modelled through the inclusion of negative feedback terms such as $k_{1}^{\prime} /\left(1+\frac{y_{3}}{s_{1}^{\prime}}\right)$ in Eq. (1). The existence of toxin $T$ in the cell reduces all protein production and decreases protein dilution by decreasing cell growth. Thus, the toxin concentration will have an inhibitory impact on the production rates of toxin, antitoxin, and on the cellular growth rate. We introduce an inhibition factor $1 /\left(b_{m}^{\prime} y_{2}+1\right)$ in Eqs. (1) - (2). The parameter $b_{m}^{\prime}$ represents the redaction of protein expression due to the presence of toxin molecules. We also assume that growth inhibition will influence the toxin degradation rate, and we introduce a factor $\left(b_{c}^{\prime} y_{2}+1\right)$ that modulates the toxin degradation rate in Eq. (2), while we assume that the degradation rate of the free antitoxin remains the same. This is in agreement with a recent finding from ${ }^{55}$ that importantly, although free antitoxin is readily degraded in vivo, antitoxin bound to toxin is protected from proteolysis, preventing release of active toxin.

However, Eqs. (1) - (3), if one includes the unknown initial conditions for the quantities $y_{1}, y_{2}, y_{3}$ at $t=0$, contain 13 adjustable parameters. Our aim is to estimate the model parameters using experimental RNA-Seq data obtained from ${ }^{41}$. These 
experimental data (10 data points for each toxin antitoxin pair) would render such an estimation problematic, since such a model is structurally unidentifiable ${ }^{56}$.

In order to reduce the number of adjustable parameters we rescale the unobserved variable $y_{3}$ by setting $y_{3}=\left(k_{2}^{\prime} / d_{3}\right) z_{3}$ and rescale the variables $y_{1}, y_{2}$ by the same factor $\beta=k_{2}^{\prime}$, i.e. by setting $y_{1}=k_{2}^{\prime} z_{2}$ and $y_{2}=k_{2}^{\prime} z_{2}$. Thus, we arrive at a system of ODEs for the rescaled variables $z_{1}, z_{2}, z_{3}$ which is:

$$
\begin{aligned}
& \frac{d z_{1}}{d t}=-d_{1} z_{1}+\frac{k_{1}}{\left(1+\frac{z_{3}}{s_{1}}\right)\left(b_{m} z_{2}+1\right)}-k_{2} z_{1} z_{2}+z_{3} \\
& \frac{d z_{2}}{d t}=-\frac{d_{2} z_{2}}{b_{c} z_{2}+1}-k_{2} z_{1} z_{2}+z_{3}+\frac{1}{\left(1+\frac{z_{3}}{s_{2}}\right)\left(b_{m} z_{2}+1\right)} \\
& \frac{d z_{3}}{d t}=-d_{3}\left(-k_{2} z_{1} z_{2}+z_{3}\right),
\end{aligned}
$$

where the new kinetic constants are related the those in Eqs. (1) - (3) by the relations $k_{1}=k_{1}^{\prime} / k_{2}^{\prime}, s_{1}=d_{3} s_{1}^{\prime} / k_{2}^{\prime}, b_{m}=$ $b_{m}^{\prime} k_{2}^{\prime}, k_{2}=k_{2}^{\prime} k_{3}, s_{2}=d_{3} s_{2}^{\prime} / k_{2}^{\prime}, b_{c}=b_{c}^{\prime} k_{2}^{\prime}$. Moreover, we assume that $z_{1}$ and $z_{2}$ at time $t=0$ are equal to zero and allow the unobserved complex concentration $z_{3}(0)$ to be equal to a constant $c_{0}$ which will be determined from the fitting of the solution of Eqs. (4) - (6) to the data. Henceforth, we will refer to the above model (Eqs. (4) - (6)) as the Z-model. The model is essentially a rescaled version of the model proposed in $^{39,40}$ with the additional assumption that the antitoxin bound to toxin is protected from proteolysis.

Our numerical investigations have shown that the Z-model (Eqs. (4) - (6)) is the simplest model able to represent the complete set of the experimental data that we have in our disposal with reasonable accuracy. Omission of any of the above basic ingredients of the model (e.g. setting $b_{m}$ and $b_{c}$ equal to zero) leads to plausible models, which may describe adequately the time evolution of the concentrations of some of the toxin-antitoxin pairs, but fail to describe the expression of the entire set. It is obvious to the reader that the Z-model and its variants that we examine in this manuscript are deterministic models. We will not deal with the important topic of investigating a stochastic variant of the Z-model through a Monte Carlo approach based on the Gillespie algorithm. Our modeling decision is based on the fact that the RNA Seq data that we will use to fit the model parameters are not single cell sequencing data. As one can see in the detailed description of the experimental data used in this study, each RNA seq "read" represents multi-cell averages on a time scale of hours. Of course for single cell RNA seq experiments a stochastic modelling approach would be more appropriate although admittedly much more difficult. There is, however, important progress in the direction of using stochastic models and the inference of parameter values from noisy data, see for example ${ }^{23}$.

For our analysis we used experimental RNA-Seq data obtained from ${ }^{41}$ (GEO accession number: GSE65244). The RNA Seq data used here are for the wild-type(wt) strain and obtained after the culture growth in rich medium during the stationary phase. The system of Eqs. (4) - (6) was solved numerically with custom code written in Python using the scipy python module ${ }^{24}$. Fitting of the numerical solutions of the ODE's was performed as part of the code using the Nelder-Mead minimization algorithm as implemented in scipy. Since the task of performing fits for all TA pairs and all model variants is quite demanding the code was parallelized using the dask.distributed python module. All numerical simulations were performed on a workstation equiped with 2 Intel Xeon Gold 6140 Processors (72 cpu cores in total).

\section{Results}

Figure 3 shows the concentrations of toxin and antitoxin for 11 known TA pairs of $E$. coli as a function of time. Symbols represent experimental RNA-Seq data obtained from ${ }^{41}$ (GEO accession number: GSE65244). The above list is exhaustive meaning that it includes all the TA pairs for which there are experimental measures in the dataset. All data have been rescaled (multiplied by the same constant $c=10^{5}$ in order to avoid numerical errors during the fitting process). Lines are the numerical solutions of the ODE system, Eqs. (1) - (3). The kinetic constants of the system were estimated so that the weighted sum of the squared differences between the experimental data and the model predictions becomes minimum. We calculate weighted least squares since we have to fit two different experimental curves simultaneously whose $y$-axis values may differ considerably. Thus, we first calculate the mean values for each curve and then the weighted sum of the squared differences. Otherwise, curves with low mean values are practically ignored during the fitting process. Thus, the lines represent the "best" fit of the model to the data. We observe a very good agreement between the model predictions and the experimental data. As mentioned above, we assume that $z_{1}$ and $z_{2}$ at time $t=0$ are equal to zero. This is a rather harsh, and probably unrealistic, condition to impose. If more data points were available the more natural and appropriate choice would be to use the RNA seq measurements of the earliest available timepoint as our initial conditions. This is indeed the approach we took in our analysis in Appendix B (Supplementary Materials). 

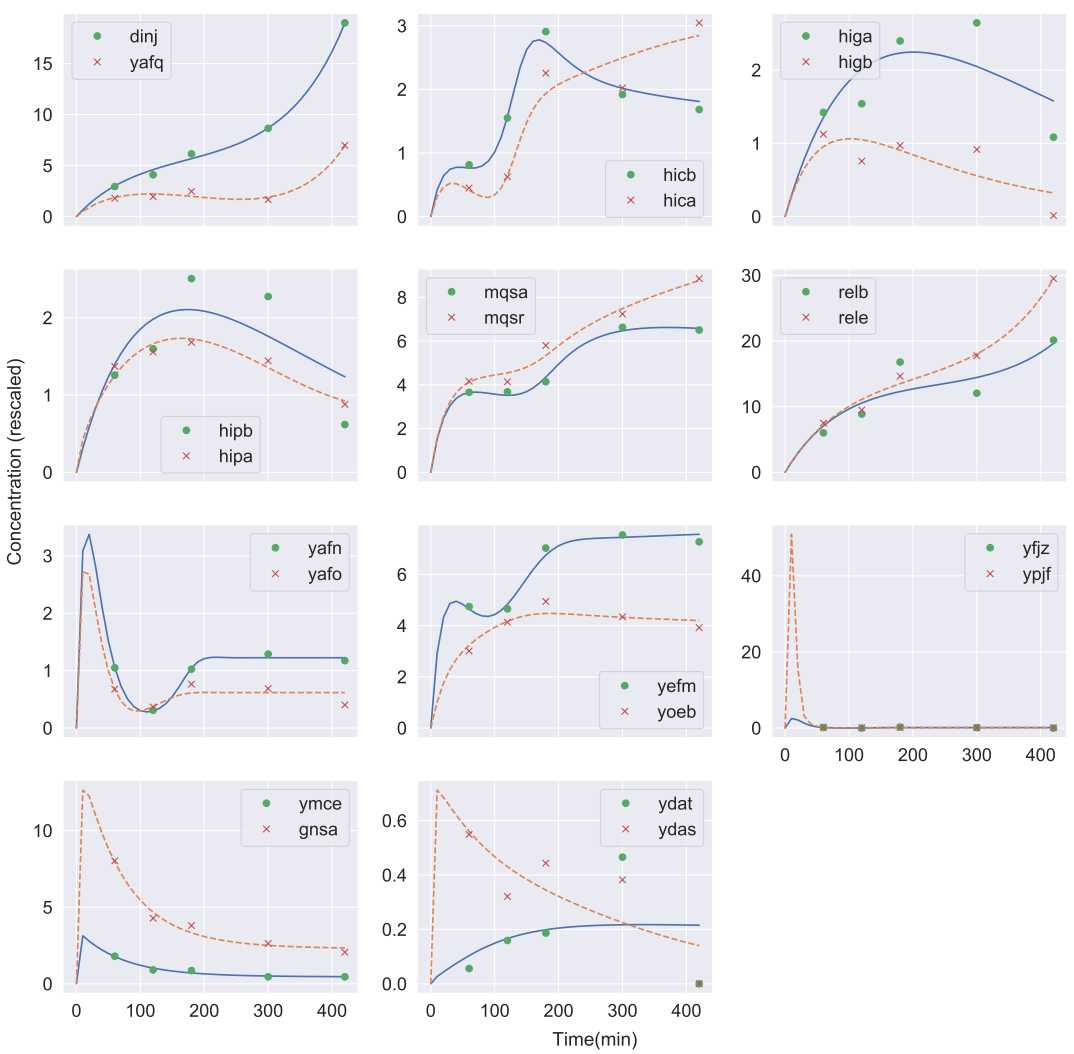

Figure 3. Toxin and antitoxin concentrations as a function of time for 11 known TA pairs of E. coli. Points represent RNA-Seq data for antitoxin (circles) and toxin (x-symbols) obtained from ${ }^{41}$. Solid lines show the result $\left(z_{1}(t)\right)$ of the numerical solution of the ODE system, Eqs. (1) - (3) for the antitoxin. Dashed lines show the corresponding variable $\left(z_{2}(t)\right)$ for the toxin.

Figure 4 shows a box plot of the model parameters estimated from the best fit of the ODE system, Eqs. (1 - 3), to the RNA-Seq data. Each box shows the "dispersion" of eleven values, one per toxin-antitoxin pair. We observe a wide distribution of parameter values across the different toxin-antitoxin pairs. This is rather common in biological systems, where the kinetic constants of various metabolic reactions can differ by several orders of magnitude. Therefore, the same underlying differential equations lead to quite different dynamics precisely due to the broad range of the kinetic constants. In Appendix A we include a detailed discussion of the estimated covariances and standard deviations of the fitting parameters (see also the attached files in supplementary materials).

Figure 5 shows in a log-linear plot the toxin, antitoxin and TA complex concentrations as a function of time for the 11 known TA pairs of $E$. coli. Solid lines show the result $y_{1}(t)$ of the numerical solution of the ODE system, Eqs. (1) - (3), for the antitoxin. Dashed lines show the corresponding variable $y_{2}(t)$ for the toxin. Dotted lines show the corresponding variable $y_{3}(t)$ for the TA complex. We observe a variety of different dynamics, but interestingly enough in all cases the complex concentration $z_{3}$ seems to be lower than that of both the toxin and the antitoxin. For the majority of cases the antitoxin concentration is higher than that of the toxin. There are, however, exceptions, namely the relB-relE, mqsR-mqsA and the ymcE-gnsA pairs. The ydaT-ydaS pair also exhibits higher toxin expression for the most part of the observation time and only at the final stage the toxin level drop below that of the antitoxin. It is also quite intriguing that the Z-model predicts expression states where the toxin is constantly quite higher than the antitoxin (e.g. $y m c E-g n s A$ ) without resorting to the mechanism of conditional cooperativity ${ }^{2,39}$, although it is quite well-established that certain TA pairs (e.g. the relB-relE pair) exhibit conditional cooperativity and, obviously, such effects are not accounted for in the Z-model.

Next, we are interested in examining simpler versions of the proposed model and assessing their ability to describe the experimental data. We compare the Z-model to 7 simpler (i.e. with less adjustable parameters) variants, which we obtain from Eqs. (4) - (6) by forcing constraints on some of the constants, i.e. by fixing their numerical value or by setting them numerically equal to other constants. We describe these simpler variants below: 


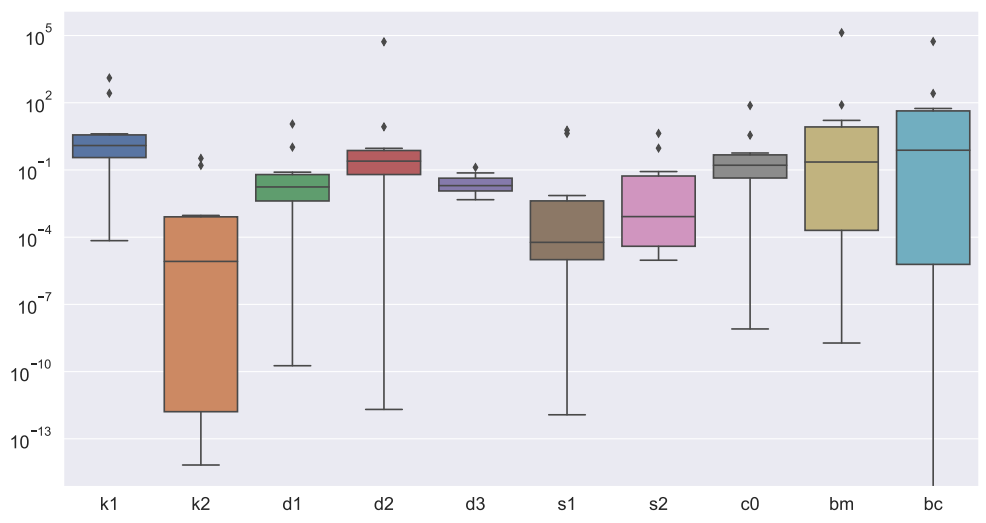

Figure 4. Box plot of the model parameters estimated from the best fit of the ODE system, Eqs.(1) - (3), to the RNA-Seq data. Each box shows the "dispersion" of eleven values, one per toxin-antitoxin pair.

- Model "s1=s2" is obtained by forcing the constants $s_{1}$ and $s_{2}$ to have the same numerical value.

- Model "s1=s2 no bm" is obtained by forcing the constants $s_{1}$ and $s_{2}$ to have the same numerical value and by dropping the $b_{m}$ constant, i.e. setting $b_{m}=0$.

- Model "s $1=\mathrm{s} 2$ no bc" is obtained forcing the constants $s_{1}$ and $s_{2}$ to have the same numerical value and by setting $b_{c}=0$.

- Model "s $1=\mathrm{s} 2$ no bm bc" is obtained by forcing the constants $s_{1}$ and $s_{2}$ to have the same numerical value and by setting both $b_{m}=0$ and $b_{c}=0$.

- Model "s1!=s2 no bm" is obtained by setting $b_{m}=0$. Note that now constants $s_{1}$ and $s_{2}$ are allowed to have different numerical values.

- Model "s1!=s2 no bc" is obtained by setting $b_{c}=0$.

- Model "no s1 s2 bm bc" is the simplest variant and is obtained from the Z-model ODEs by setting $s_{1}=1, s_{2}=1, b_{m}=$ $0, b_{c}=0$.

Models, where the parameter $b_{m}$ is identically zero, do not take into account the reduction of protein expression due to the existence of toxin, while variants, where the parameter $b_{c}$ is identically zero, ignore the effect of growth inhibition. Fig 6 shows the minimum values of the objective function (i.e. the sum of weighted squared differences between model predictions and the experimental data) for all toxin-antitoxin pairs and for the 7 model variants described above.

The objective function of the Full Z-model is always lower than that of the variants, as expected. We should also mention that the algorithms (basinhopping in combination with a local Nelder-Mead algorithm) used for the minimization of the objective function are guaranteed to find local, not global, minima. Although we have performed a rather extensive search of the parameter space, there is always the chance that there are sets of parameters that will lead to lower values of the objective function than those reported here. We see that there are toxin-antitoxin pairs for which simpler variants are capable of fitting the data with results comparable to those of the Full Z-model. However, the Full Z-model is the appropriate choice if one wants to describe the expression of the entire set of TA pairs.

Since we want to compare models with different numbers of parameters, it might be plausible to examine two widely used model selection criteria, the Akaike Information Criterion (AIC) and the Bayesian Information Criterion (BIC) for the Full Z-model and its seven variants. These are calculated as follows:

$$
\begin{aligned}
& \text { aic }=N \ln \left(\chi^{2} / N\right)+2 N_{v} \\
& \text { bic }=N \ln \left(\chi^{2} / N\right)+\ln (N) N_{v},
\end{aligned}
$$

where $\chi^{2}$ stands for the sum of the squares of the residuals (i.e., the objective function discussed above), $N$ is the number of data points (common for all model variants) and $N_{v}$ is the number of adjustable parameters for each model. $N_{v}$ is different for 

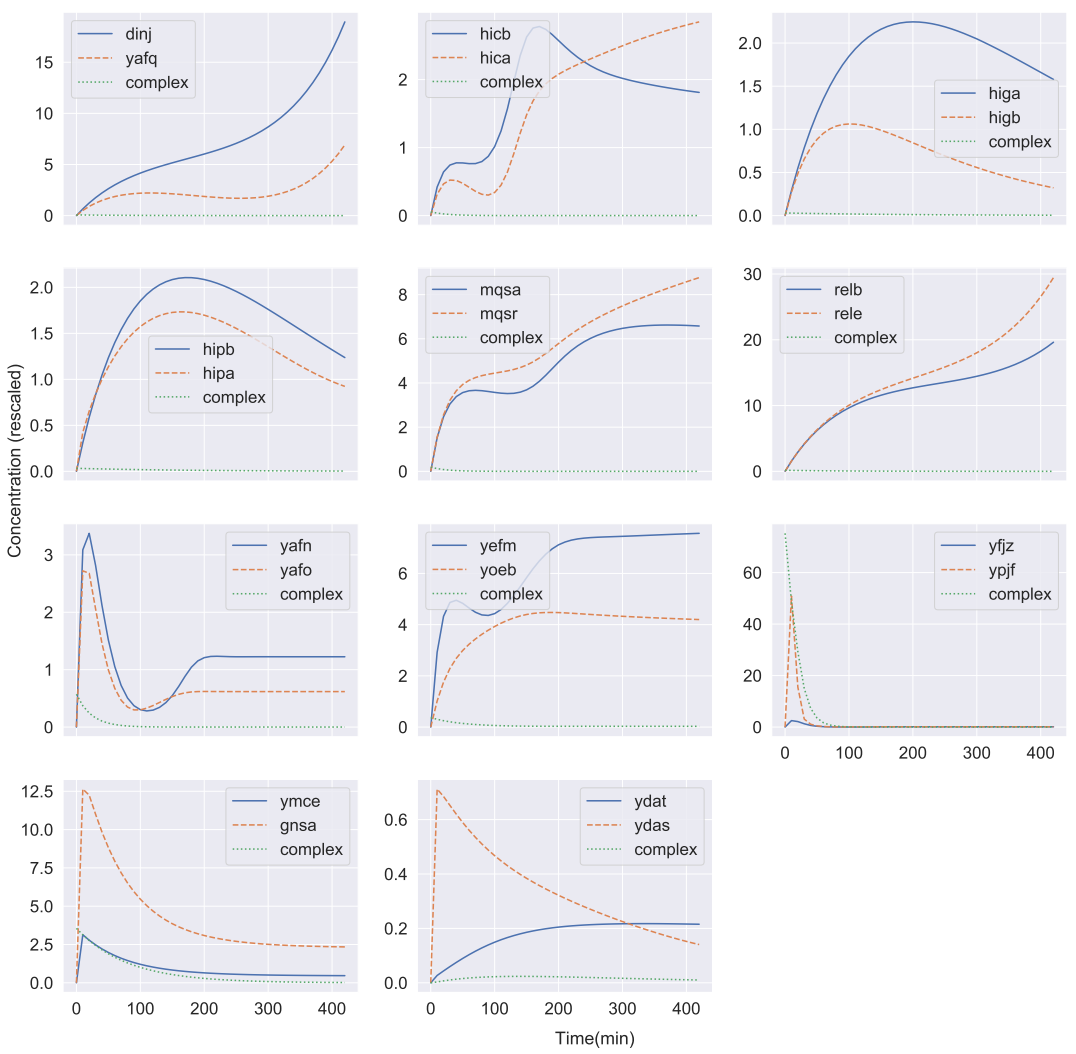

Figure 5. Toxin, antitoxin and TA complex concentrations as a function of time for 11 known TA pairs of E.coli. Solid lines show the result $z_{1}(t)$ of the numerical solution of the ODE system, Eqs.(1) - (3), for the antitoxin. Dashed lines show the corresponding variable $z_{2}(t)$ for the toxin. Dotted lines show the corresponding variable $z_{3}(t)$ for the TA complex. Notice that the $y$-axis has a logarithmic scale.

each variant. The full Z-model has the highest value, i.e. $N_{v}=10$. The most appropriate model is considered to be the one with the lower AIC or BIC value since both these criteria penalize the a large $N_{v}$ number and reward a low objective function. Generally, the Bayesian information criterion is considered the most conservative of the two statistics. Figure 7 shows the AIC and BIC for the "collective" description of the TA gene expression set, i.e. when we describe the complete set of TA-pair with $N=10 * 11=110$ data points and $\chi^{2}$ is the sum of the objective functions of all the TA pairs.

Finally, it is helpful to compare the values of the constants that we obtained from the minimal ODE model for the different TA pairs. To this end we may view them as a "vector" characterizing the TA pair and we use an unsupervised learning method, namely a Principal Component Analysis (PCA), a statistical procedure that uses an orthogonal transformation to convert a set of observations of possibly correlated variables into a set of values of linearly uncorrelated variables called principal components ${ }^{58}$. PCA is routinely applied to experimental measurments directly for reasons of dimensionality reduction. Using PCA, however, to interpret the parameters of a deterministic ODE model consists a novel approach which has been recently used to interpret the parameters of a fractal kinetics SI model of Covid-19 spreading $^{59}$. Figure 8 shows a plot of the two largest PCA components.

Typically in a PCA plot we try to identify clusters and perceive them as an indication of similar underlying causal behavior. For cluster identification, to avoid subjectivity, we applied a clustering identification algorithm i.e. DBSCAN with parameter eps $=0.8^{60}$. For DBSCAN the number of clusters is not predefined but decided by the algorithm. Here, the clustering algorithm has identified one cluster of 7 TA pairs, namelydinJ-yafQ, relB-relE, yafN-yafO, higA-higB, hipB-hipA, hicB-hicA, and $m q s A-m q s R$, which form a large central cluster, and four outliers i.e. the three pairs yefM-yoeB, ydaT-ydaS, and ymcE-gnsA, which have a negative PC2 component, and yfjZ-ypjF with relatively large PC1 and PC2 values.

In Table 1 we summarize this distinction between a main cluster and several outliers, together with the associated functional classification of the TA pairs. This distinction can serve as a starting point for comparing this statistical result with the wealth 


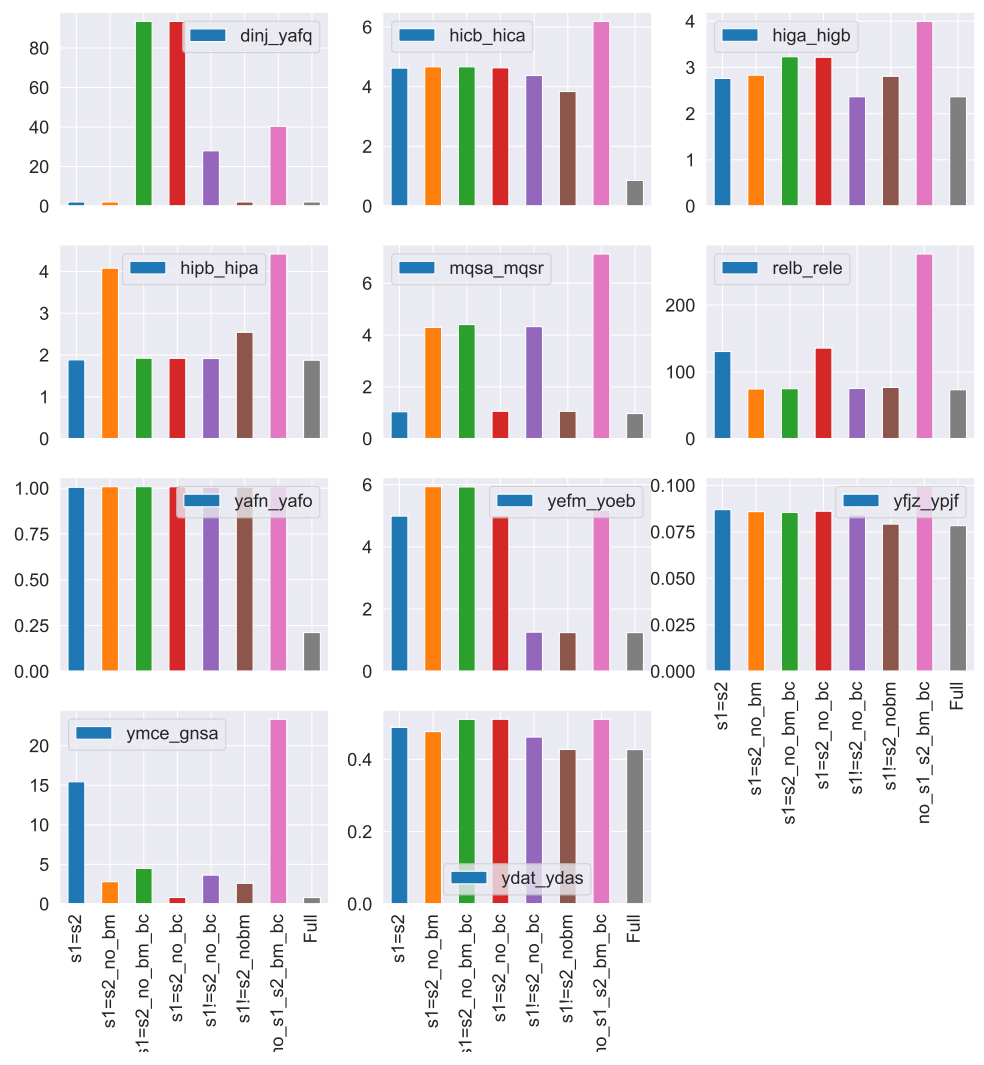

Figure 6. The values of the objective function for all toxin-antitoxin pairs and for 7 model variants. The Z-model defined by Eqs. (4) - (6) is marked with the label "Full" in the x-axis.

of biological information available for each of these toxin-antitoxin modules. For the toxin-antitoxin module hipB-hipA for example, the mode of action has been debated over the last years ${ }^{62,63}$, but is still not clear ${ }^{1}$. The similarity of estimated parameters to higA-higB, hicB-hicA and other members of the main cluster may be seen as evidence of a functional classification of this TA system as RNA interferases and guide further attempts of functional elucidation, in particular a better understanding of superfamilies of type-II TA systems ${ }^{64}$.

Table 1. Identified clusters and their functional classification.

$\begin{array}{llc}\begin{array}{l}\text { Operon } \\ \text { Main cluster }\end{array} & \text { Functional classification } & \text { Refe } \\ \text { dinJ-yafQ } & \text { Ribosome-dependent RNA interferases } & 1,67 \\ \text { relB-relE } & \text { Ribosome-dependent RNA interferases } & 1,68 \\ \text { yafN-yafO } & \text { Ribosome-dependent RNA interferases } & 1,36 \\ \text { higA-higB } & \text { Ribosome-dependent RNA interferases } & 1,65 \\ \text { hipB-hipA } & \text { Unknown (involved in persistence) } & 1,62 \\ \text { hicB-hicA } & \text { Ribosome-independent RNA interferases } & 1,66 \\ \text { mqsA-mqsR } & \text { Ribosome-independent RNA interferases } & 1,25 \\ \text { Outliers } & & \\ \text { yefM-yoeB } & \text { Ribosome-dependent RNA interferases } & 1 \\ \text { ydaT-ydaS } & \text { Unknown } & 1 \\ \text { ymcE-gnsA } & \text { Inhibitor of phospholipid synthesis } & 1,52 \\ \text { yfjZ-ypjF } & \text { Inhibitors of cell division } & 1\end{array}$




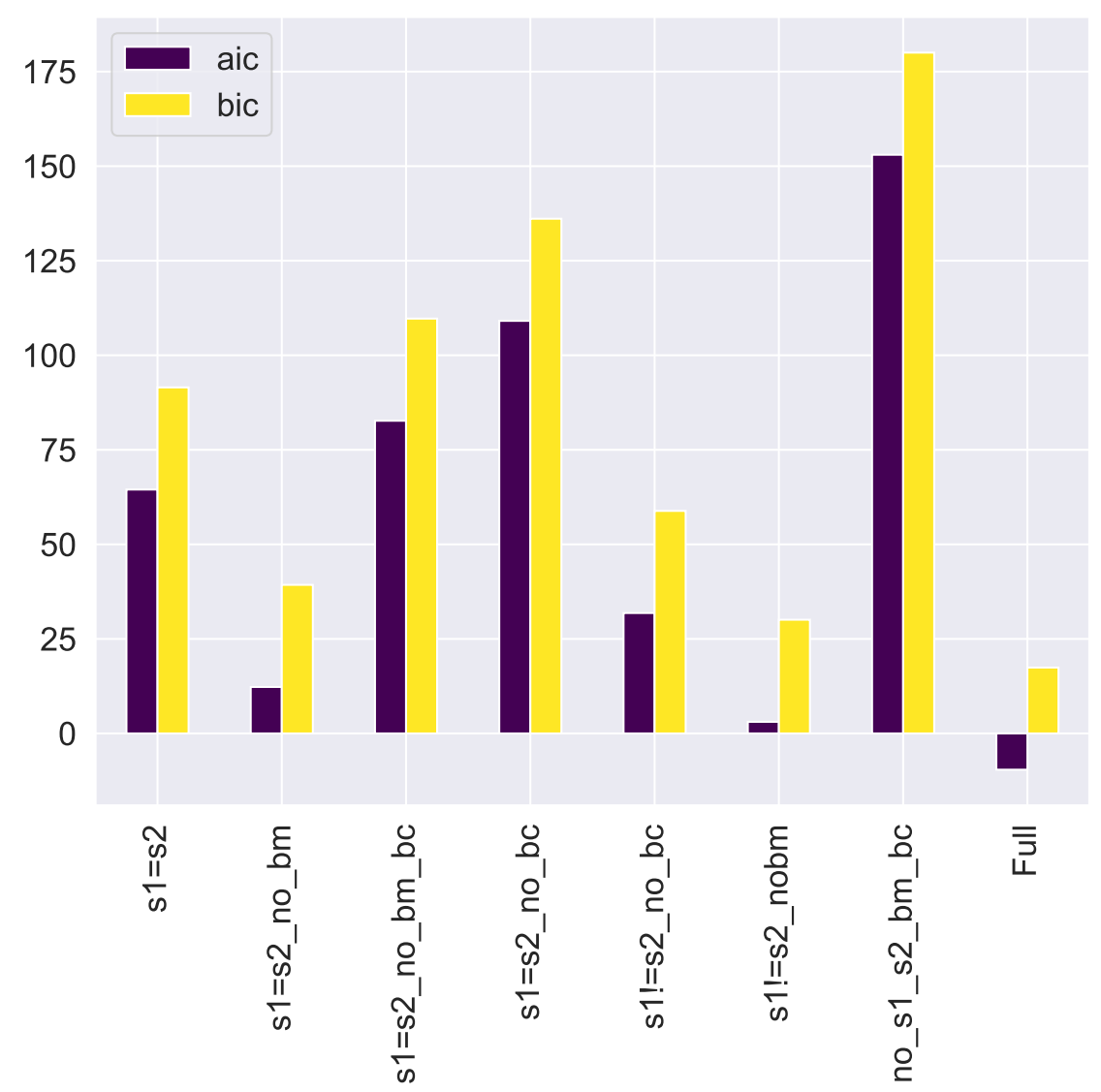

Figure 7. Collective Akaike Information Criterion (AIC) and Baeysian Information Criterion (BIC) for the Full Z-model and its seven variants. The Full Z-model has clearly the lowest AIC and BIC among all variants studied.

Appendix B (Supplementary Materials) contains the results for another time-resolved gene expression data set, namely the data from ${ }^{69}$ which are available at GEO (accession number: GSE131992).

In Appendix C (Supplementary Materilas), we present in tabular form the biological information relevant to the members of the clusters identified in Fig. 7 as obtained from The Universal Protein Resource (UniProt), a comprehensive resource for protein sequence and annotation data (https://www.uniprot.org).

\section{Conclusions}

We have proposed a minimal model that is able to capture the dynamics of toxin-antitoxin systems in E. coli and agrees with experimental high-throughput RNA-Seq data reasonably well. We find that a minimal acceptable model of toxin-antitoxin regulation should at least include a negative feedback loop through a TA pair formation and the effect of toxin induced growth modulation. Despite the obvious over-simplifications of the model, e.g. we study each toxin-antitoxin pair in isolation, and we do not account for the influence on cell growth due to the remaining toxin proteins, the model is able to replicate a variety of experimental curves.

With the availability of more time-resolved high-quality gene expression data, the description of time courses of systemic components with the help of simple mathematical models can provide an important instrument for the interpretation of such high-throughput data and thus bridge the gap between Theoretical Biology, Statistical Physics and Systems Biology.

\section{Author contributions statement}

K.K, M-T.H designed research, K.K. performed research, K.K, M-T.H analyzed the results, K.K, M-T.H wrote the manuscript. 


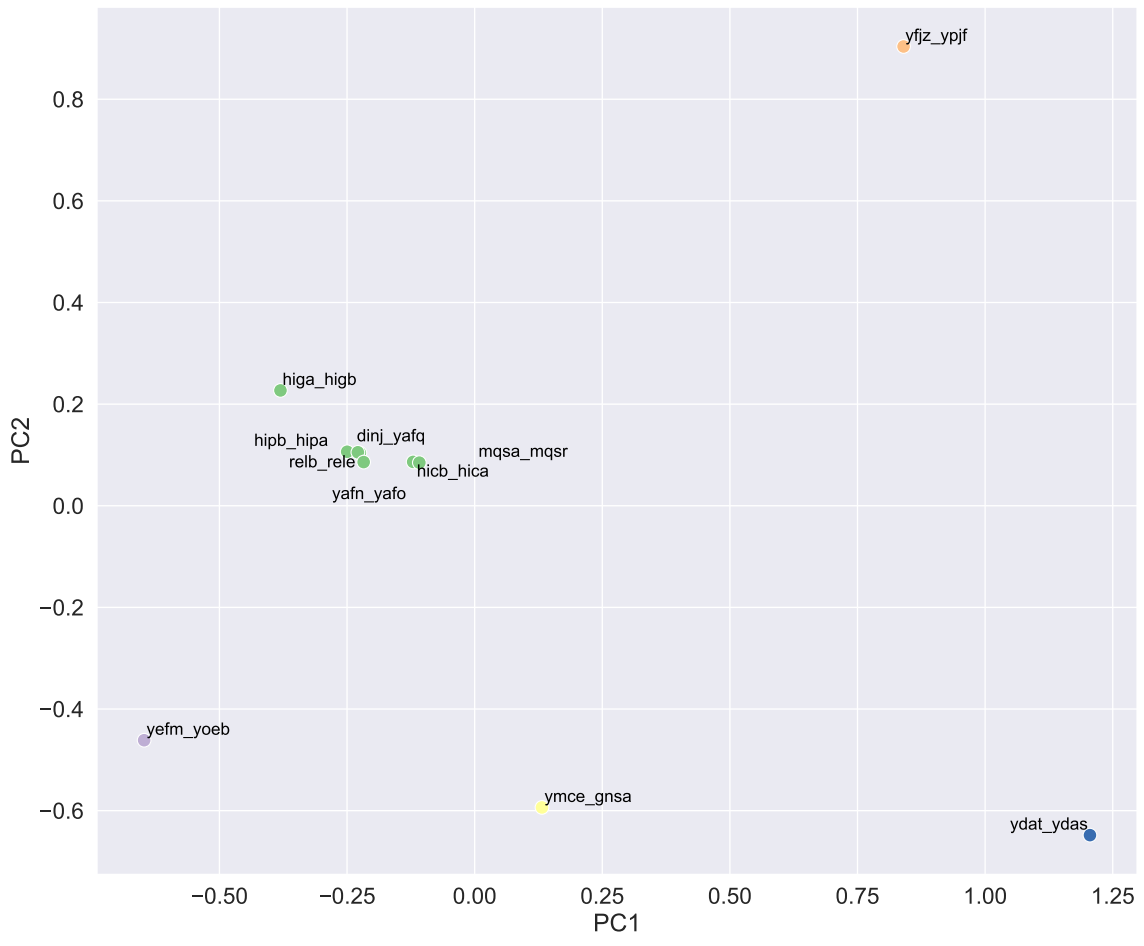

Figure 8. Plot of the largest $(P C 1)$ vs. the second largest $(P C 2)$ principal components. A distinction between one main cluster and a set of outliers can be discerned: Central Cluster dinJ-yafQ, relB-relE, yafN-yafO, higA-higB, hipB-hipA, hicB-hicA, and mqsA-mqsR. Outliers yefM-yoeB, ydaT-ydaS, ymcE-gnsA, and yfjZ-ypjF.

\section{Additional information}

Competing interests The authors declare no competing interests

Data availability The datasets analysed and the custom code used during the current study are available from the corresponding author on reasonable request. 


\section{References}

1. Yamaguchi Y, Inouye M. 2011 Regulation of growth and death in Escherichia coli by toxin-antitoxin systems. Nature Reviews Microbiology 9, 779-790.

2. Page R, Peti W. 2016 Toxin-antitoxin systems in bacterial growth arrest and persistence. Nature Chemical Biology 12, 208-214.

3. Pandey DP, Gerdes K. 2005 Toxin-antitoxin loci are highly abundant in free-living but lost from host-associated prokaryotes. Nucleic Acids Research 33, 966-976.

4. Balaban NQ, Merrin J, Chait R, Kowalik L, Leibler S. 2004 Bacterial persistence as a phenotypic switch. Science 305, $1622-1625$.

5. Harms A, Brodersen DE, Mitarai N, Gerdes K. 2018 Toxins, targets, and triggers: an overview of toxin-antitoxin biology. Molecular Cell 70, 768-784.

6. Thisted T, Sørensen N, Wagner E, Gerdes K. 1994 Mechanism of post-segregational killing: Sok antisense RNA interacts with Hok mRNA via its $5^{\prime}$-end single-stranded leader and competes with the $3^{\prime}$-end of Hok mRNA for binding to the mok translational initiation region. The EMBO Journal 13, 1960-1968.

7. Gerdes K, Nielsen A, Thorsted P, Wagner EGH. 1992 Mechanism of killer gene activation. Antisense RNA-dependent RNase III cleavage ensures rapid turn-over of the stable hok, srnB and pndA effector messenger RNAs. Journal of Molecular Biology 226, 637-649.

8. Brantl S, Jahn N. 2015 sRNAs in bacterial type I and type III toxin-antitoxin systems. FEMS Microbiology Reviews 39, 413-427.

9. Pedersen K, Gerdes K. 1999 Multiple hok genes on the chromosome of Escherichia coli. Molecular Microbiology 32, 1090-1102.

10. Brown JM, Shaw KJ. 2003 A novel family of Escherichia coli toxin-antitoxin gene pairs. Journal of Bacteriology 185, 6600-6608.

11. Masuda H, Tan Q, Awano N, Wu KP, Inouye M. 2012 YeeU enhances the bundling of cytoskeletal polymers of MreB and FtsZ, antagonizing the CbtA (YeeV) toxicity in Escherichia coli. Molecular Microbiology 84, 979-989.

12. Wang X, Lord DM, Cheng HY, Osbourne DO, Hong SH, Sanchez-Torres V, Quiroga C, Zheng K, Herrmann T, Peti W et al.. 2012 A new type V toxin-antitoxin system where mRNA for toxin GhoT is cleaved by antitoxin GhoS. Nature Chemical Biology 8, 855.

13. Wang X, Lord DM, Hong SH, Peti W, Benedik MJ, Page R, Wood TK. 2013 Type II toxin/antitoxin MqsR/MqsA controls type V toxin/antitoxin GhoT/GhoS. Environmental Microbiology 15, 1734-1744.

14. Aakre CD, Phung TN, Huang D, Laub MT. 2013 A bacterial toxin inhibits DNA replication elongation through a direct interaction with the $\beta$ sliding clamp. Molecular Cell 52, 617-628.

15. Balaban NQ, Helaine S, Lewis K, Ackermann M, Aldridge B, Andersson DI, Brynildsen MP, Bumann D, Camilli A, Collins JJ, Dehio C, Fortune S, Ghigo JM, Hardt WD, Harms A, Heinemann M, Hung DT, Jenal U, Levin BR, Michiels J, Storz G, Tan MW, Tenson T, Van Melderen L, Zinkernagel A. 2019 Definitions and guidelines for research on antibiotic persistence. Nature Reviews Microbiology 17, 441-448.

16. Sneppen K, Micheelsen MA, Dodd IB. 2008 Ultrasensitive gene regulation by positive feedback loops in nucleosome modification. Molecular Systems Biology.

17. Fasani RA, Savageau MA. 2013 Molecular mechanisms of multiple toxin-antitoxin systems are coordinated to govern the persister phenotype. Proceedings of the National Academy of Sciences 110, E2528-E2537.

18. Fraikin N, Goormaghtigh F, Van Melderen L. 2020 Type II toxin-antitoxin systems: evolution and revolutions. Journal of Bacteriology 202.

19. Leplae R, Geeraerts D, Hallez R, Guglielmini J, Dreze P, Van Melderen L. 2011 Diversity of bacterial type II toxin-antitoxin systems: a comprehensive search and functional analysis of novel families. Nucleic Acids Research 39, 5513-5525.

20. Ghafourian S, Raftari M, Sadeghifard N, Sekawi Z. 2014 Toxin-antitoxin systems: classification, biological function and application in biotechnology. Curr Issues Mol Biol 16, 9-14.

21. Fasani RA, Savageau MA. 2015 Unrelated toxin-antitoxin systems cooperate to induce persistence. Journal of The Royal Society Interface 12, 20150130. 
22. Gerdes K. 2016 Hypothesis: type I toxin-antitoxin genes enter the persistence field - a feedback mechanism explaining membrane homoeostasis. Philosophical Transactions of the Royal Society B: Biological Sciences 371, 20160189.

23. Cao Z., Grima R. 2019 Accuracy of parameter estimation for auto-regulatory transcriptional feedback loops from noisy data Journal of The Royal Society Interface 16, 20180967.

24. Virtanen P. et al. 2020 SciPy 1.0: Fundamental Algorithms for Scientific Computing in Python Nature Methods 16, 261-272

25. Kedzierska B, Hayes F. 2016 Emerging roles of toxin-antitoxin modules in bacterial pathogenesis. Molecules 21, 790.

26. Massey SE, Mishra B. 2018 Origin of biomolecular games: deception and molecular evolution. Journal of the Royal Society Interface 15, 20180429.

27. Ruangprasert A, Maehigashi T, Miles SJ, Giridharan N, Liu JX, Dunham CM. 2014 Mechanisms of toxin inhibition and transcriptional repression by Escherichia coli DinJ-YafQ. Journal of Biological Chemistry 289, 20559-20569.

28. Takagi H, Kakuta Y, Okada T, Yao M, Tanaka I, Kimura M. 2005 Crystal structure of archaeal toxin-antitoxin RelE-RelB complex with implications for toxin activity and antitoxin effects. Nature Structural \& Molecular Biology 12, 327.

29. Zhang Y, Zhu L, Zhang J, Inouye M. 2005 Characterization of ChpBK, an mRNA interferase from Escherichia coli. Journal of Biological Chemistry 280, 26080-26088.

30. Motiejūnaite R, Armalytė J, Markuckas A, Sužiedèlienė E. 2007 Escherichia coli dinJ-yafQ genes act as a toxin-antitoxin module. FEMS Microbiology Letters 268, 112-119.

31. Prysak MH, Mozdzierz CJ, Cook AM, Zhu L, Zhang Y, Inouye M, Woychik NA. 2009 Bacterial toxin YafQ is an endoribonuclease that associates with the ribosome and blocks translation elongation through sequence-specific and frame-dependent mRNA cleavage. Molecular Microbiology 71, 1071-1087.

32. Kamada K, Hanaoka F. 2005 Conformational change in the catalytic site of the ribonuclease YoeB toxin by YefM antitoxin. Molecular Cell 19, 497-509.

33. Zhang Y, Inouye M. 2009 The inhibitory mechanism of protein synthesis by YoeB, an Escherichia coli toxin. Journal of Biological Chemistry 284, 6627-6638.

34. Keren I, Shah D, Spoering A, Kaldalu N, Lewis K. 2004 Specialized persister cells and the mechanism of multidrug tolerance in Escherichia coli. Journal of Bacteriology 186, 8172-8180.

35. Korch SB, Henderson TA, Hill TM. 2003 Characterization of the hipA7 allele of Escherichia coli and evidence that high persistence is governed by (p) ppGpp synthesis. Molecular Microbiology 50, 1199-1213.

36. Zhang Y, Yamaguchi Y, Inouye M. 2009 Characterization of YafO, an Escherichia coli toxin. Journal of Biological Chemistry 284, 25522-25531.

37. Brown BL, Grigoriu S, Kim Y, Arruda JM, Davenport A, Wood TK, Peti W, Page R. 2009 Three dimensional structure of the MqsR: MqsA complex: a novel TA pair comprised of a toxin homologous to RelE and an antitoxin with unique properties. PLoS Pathogens 5, e1000706.

38. Vandervelde A, Loris R, Danckaert J, Gelens L. 2016 Computational methods to model persistence. In Michiels J, Fauvart M, editors, Methods in Molecular Biology, Methods in Molecular Biology, vol. 1333, pp. 207-240. New York, NY: Springer New York.

39. Cataudella I, Sneppen K, Gerdes K, Mitarai N. 2013 Conditional cooperativity of toxin - antitoxin regulation can mediate bistability between growth and dormancy. PLoS Computational Biology 9, e1003174.

40. Cataudella I, Trusina A, Sneppen K, Gerdes K, Mitarai N. 2012 Conditional cooperativity in toxin-antitoxin regulation prevents random toxin activation and promotes fast translational recovery. Nucleic Acids Research 40, 6424-6434.

41. Beber ME, Sobetzko P, Muskhelishvili G, Hütt MT. 2016 Interplay of digital and analog control in time-resolved gene expression profiles. EPJ Nonlinear Biomedical Physics 4, 8.

42. Gelens L, Hill L, Vandervelde A, Danckaert J, Loris R. 2013 A general model for toxin-antitoxin module dynamics can explain persister cell formation in E. coli. PLoS Comput Biol 9, e1003190.

43. Nikolic N, Bergmiller T, Vandervelde A, Albanese TG, Gelens L, Moll I. 2018 Autoregulation of mazEF expression underlies growth heterogeneity in bacterial populations. Nucleic acids research 46, 2918-2931.

44. Tian C, Semsey S, Mitarai N. 2017 Synchronized switching of multiple toxin-antitoxin modules by (p) ppGpp fluctuation. Nucleic Acids Research 45, 8180-8189. 
45. Shen-Orr SS, Milo R, Mangan S, Alon U. 2002 Network motifs in the transcriptional regulation network of Escherichia coli. Nature Genetics 31, 64.

46. Alon U. 2007 Network motifs: theory and experimental approaches. Nature Reviews Genetics 8, 450-461.

47. Marr C, Geertz M, Hütt MT, Muskhelishvili G. 2008 Dissecting the logical types of network control in gene expression profiles. BMC systems biology $2,18$.

48. Travers A, Muskhelishvili G, Thompson J. 2012 DNA information: from digital code to analogue structure. Phil. Trans. R. Soc. A 370, 2960-2986.

49. Sonnenschein N, Geertz M, Muskhelishvili G, Hütt MT. 2011 Analog regulation of metabolic demand. BMC Syst Biol 5, 40.

50. Kosmidis K, Jablonski KP, Muskhelishvili G, Hütt MT. 2020 Chromosomal origin of replication coordinates logically distinct types of bacterial genetic regulation. NPJ Systems Biology and Applications 6, 1-9.

51. Fang X, Sastry A, Mih N, Kim D, Tan J, Yurkovich JT, Lloyd CJ, Gao Y, Yang L, Palsson BO. 2017 Global transcriptional regulatory network for Escherichia coli robustly connects gene expression to transcription factor activities. Proceedings of the National Academy of Sciences 114, 10286-10291.

52. Wei, Y., Zhan, L., Gao, Z., Privé, G. G., and Dong, Y. (2015). Crystal structure of GnsA from Escherichia coli. Biochemical and Biophysical Research Communications, 462(1):1-7.

53. Vet S, Vandervelde A, Gelens L. 2019 Excitable dynamics through toxin-induced mRNA cleavage in bacteria. PloS One 14.

54. Himeoka Y, Mitarai N. 2019 Modeling slow-processing of toxin messenger RNAs in type-I toxin-antitoxin systems: post-segregational killing and noise filtering. Physical Biology 16, 026001.

55. LeRoux M, Culviner PH, Liu YJ, Littlehale ML, Laub MT. 2020 Stress Can Induce Transcription of Toxin-Antitoxin Systems without Activating Toxin. Molecular Cell.

56. DiStefano III J. 2015 Dynamic systems biology modeling and simulation. Academic Press.

57. Yamaguchi, Y., Park, J.-H., and Inouye, M. (2009) MqsR, a crucial regulator for quorum sensing and biofilm formation, is a GCU-specific mRNA interferase in Escherichia coli. Journal of Biological Chemistry, 284(42):28746-28753.

58. Jolliffe I. 2002 Principal Component Analysis. Springer; 2nd edition.

59. Kosmidis K., Macheras P. 2020 A fractal kinetics SI model can explain the dynamics of COVID-19 epidemics PloS One 15, e0237304.

60. Schubert E, Sander J, Ester M, Kriegel HP, Xu X. 2017 DBSCAN revisited, revisited: why and how you should (still) use DBSCAN. ACM Transactions on Database Systems (TODS) 42, 1-21.

61. Schureck MA, Meisner J, Hoffer ED, Wang D, Onuoha N, Ei Cho S, Lollar III P, Dunham CM. 2019 Structural basis of transcriptional regulation by the HigA antitoxin. Molecular Microbiology 111, 1449-1462.

62. Germain E, Castro-Roa D, Zenkin N, Gerdes K. 2013 Molecular mechanism of bacterial persistence by HipA. Molecular Cell 52, 248-254.

63. Hansen S, Vulić M, Min J, Yen TJ, Schumacher MA, Brennan RG, Lewis K. 2012 Regulation of the Escherichia coli HipBA toxin-antitoxin system by proteolysis. PloS One 7.

64. Guglielmini J, Van Melderen L. 2011 Bacterial toxin-antitoxin systems: Translation inhibitors everywhere. Mobile Genetic Elements 1, 283-306.

65. Hurley JM, Woychik NA. 2009 Bacterial toxin HigB associates with ribosomes and mediates translation-dependent mRNA cleavage at A-rich sites. Journal of Biological Chemistry 284, 18605-18613.

66. Unterholzner SJ, Poppenberger B, Rozhon W. 2013 Toxin-antitoxin systems: biology, identification, and application. Mobile Genetic Elements 3, e26219.

67. Armalytė J, Jurènaitė M, Beinoravičiūtė G, Teišerskas J, Sužiedèlienė E. 2012 Characterization of Escherichia coli dinJ-yafQ toxin-antitoxin system using insights from mutagenesis data. Journal of Bacteriology 194, 1523-1532.

68. Gerdes K. 2013 pp. 69-92. In Type II Toxin-Antitoxins Loci: The relBE Family, pp. 69-92. Berlin, Heidelberg: Springer Berlin Heidelberg.

69. Lempp M, Farke N, Kuntz M, Freibert SA, Lill R, Link H. 2019 Systematic identification of metabolites controlling gene expression in E. coli. Nature communications 10, 1-9. 


\section{Supplementary Files}

This is a list of supplementary files associated with this preprint. Click to download.

- SRToxinAntitoxinsupplementarymaterials.pdf 